\title{
Multiple cystic lesions after treatment for pulmonary cryptococcosis
}

\author{
Tomoyo Taketa ${ }^{1}$ and Takahito Nakamura ${ }^{1}$ \\ ${ }^{1}$ Hoshigaoka Iryo Center
}

May 17, 2021

\begin{abstract}
Pulmonary cryptococcosis presents as various radiological manifestations depending on the immunological status of the host. The most common chest radiographic findings include single or multiple nodules. Herein, we present a rare case of pulmonary cryptococcosis in a patient with rheumatoid arthritis presenting as multiple cystic lesions that enlarged after treatment.
\end{abstract}

\section{Introduction}

Pulmonary cryptococcosis caused by Cryptococcus neoformans occurs in immunocompetent hosts as well as in immunocompromised patients ${ }^{1}$.

The condition presents with various imaging findings, especially in immunocompromised hosts ${ }^{2}$. Pulmonary nodules/masses, either solitary or multiple, are the most common radiological findings, which are sometimes associated with cavitation, halo sign, and air bronchogram. In addition, it may present with consolidation, ground-glass opacity, linear opacity, and lymphadenopathy ${ }^{1}$.

In most cases, lesions noted on computed tomography (CT) improve after antifungal treatment; however, the resolution is usually gradual and incomplete among the non-surgical cases $^{3}$.

Here, we report an extremely rare case of pulmonary cryptococcosis presenting as multiple cystic lesions that enlarged after antifungal treatment.

\section{Case presentation}

A 77-year-old man with a history of rheumatoid arthritis (RA) for 15 years was admitted to our department due to organizing pneumonia secondary to RA. His symptoms and radiological findings improved after initiating corticosteroid therapy (500 $\mathrm{mg}$ /day methylprednisolone for three consecutive days, followed by $1 \mathrm{mg} / \mathrm{kg}$ /day prednisolone), which was gradually tapered in the outpatient department. He had a medical history of diabetes mellitus. He had smoked 20 cigarettes a day for approximately 40 years. He had been treated with iguratimod $(25 \mathrm{mg} /$ day $)$ and prednisolone $(3 \mathrm{mg} /$ day $)$ for RA. Approximately 1 year after discharge, he complained of cough and sputum production. A physical examination revealed no acute distress, with a blood pressure of $135 / 67 \mathrm{mmHg}$, heart rate of $87 \mathrm{bpm}$, body temperature of $37.1^{\circ} \mathrm{C}$, and respiratory rate of $20 / \mathrm{min}$. A neurological examination showed no abnormalities. Laboratory tests showed elevated C-reactive protein level and renal dysfunction. The levels of serum $\beta$-D glucan, MPO-ANCA, and PR3-ANCA were normal. Tests for Aspergillus antigens, interferon-gamma release assays for Mycobacterium tuberculosis, and tests for anti-Mycobacteria antibody were negative (Table 1). CT showed multiple cavities in the right lung (Figure 1A). Sputum culture resulted in the isolation of C. neoformans, and tests for serum Cryptococcus antigens were positive (antigen titer: 1:64). Bronchoscopy was performed, and culture of the bronchial lavage fluid was found to be positive for $C$. neoformans. Therefore, the patient was diagnosed with pulmonary cryptococcosis. Lumbar puncture was performed, which revealed clear cerebrospinal fluid with a normal cell count and normal protein and glucose levels. Tests for the cryptococcal antigen in the cerebrospinal fluid were negative. The patient received fluconazole orally (400 mg/day), but the treatment 
was interrupted due to drug eruption 1 month later. Subsequently, he was treated with oral administration of itraconazole $(200 \mathrm{mg} /$ day $)$. After the initiation of this treatment, his symptoms and inflammatory responses in laboratory tests improved. CT showed that the walls of the lesions became thin and they coalesced with each other (Figure 1B). Six months after the initiation of treatment, he complained of exertional dyspnea. CT revealed enlarged multiple cysts, which may have caused his symptoms (Figure 1C). The cryptococcal antigen titer was 1:4, and no increase in the antigen titer was observed thereafter; the treatment was completed in 9 months.

Although no significant increase in the size of cystic lesions was observed after the end of treatment, exertional dyspnea of the patient persisted and the risk of pneumothorax was high; therefore, the patient is being followed up carefully.

\section{Discussion}

Pulmonary cryptococcosis presenting with multiple cystic lesions is a rare phenomenon. Moreover, in our patient, the lesions enlarged after the initiation of treatment although cryptococcosis was controlled. Our experience shows that pulmonary cryptococcosis might present with multiple cystic lesions during the course of treatment. Moreover, new clinical symptoms such as exertional dyspnea might be observed and careful follow-up is required after treatment.

Cryptococcosis occurs in both immunocompetent and immunocompromised individuals ${ }^{2}$. The radiographic features of pulmonary cryptococcosis are varied, depending on the immunological status of the host ${ }^{1}$. Patients with RA show immunodeficiency due to drug treatment and autoimmune inflammation, as well as variable CT findings and distribution in pulmonary cryptococcosis ${ }^{4,5}$.

In pulmonary cryptococcosis, cavitation is occasionally detected on CT, especially in immmunocompromised patients $^{1}$. There have been few case reports of pulmonary cryptococcosis with multiple cystic lesions. A lung cyst is defined as any round circumscribed space surrounded by an epithelial or fibrous wall of variable thickness that usually measures less than $3 \mathrm{~mm}$. It can be distinguished from a cavity in which the wall thickness is greater than $3 \mathrm{~mm}^{6}$. The radiographic findings in the present case presented as cysts. Takata et al. reported a case of secondary pulmonary cryptococcosis presenting with multiple cystic shadows in a patient with RA, with size reduction of cystic lesions after the initiation of treatment ${ }^{7}$. However, in our case, the cystic lesions became coalesced with each other, taking on a thin-walled state, and became enlarged, despite improvement in clinical manifestation and antigen titer. A previous report has indicated that the radiologic resolution of pulmonary cryptococcosis following antifungal therapy is usually gradual and incomplete ${ }^{3}$. Residual abnormalities, including bronchiectasis, cavitation, and/or fibrotic scars, are frequently observed, most notably on $\mathrm{CT}^{3}$.

We speculated that the mechanism of the formation and development of cysts in this case refer to the etiology of other diseases presenting with multiple cystic lesions. Pathologically, most nodules and masses seen on radiographic patterns in pulmonary cryptococcosis are characterized by granulomatous reactions. Langerhans cell histiocytosis (LCH) is characterized by the infiltration of Langerhans cells mainly in the lungs, leading to the activation of an inflammatory process with potential dysfunction in the affected sites $^{8}$. Granuloma formation in LCH is similar to the distribution of pulmonary cryptococcosis ${ }^{9}$. The pathogenesis of pulmonary cysts in LCH is speculated to be peribronchiolar distribution of granulomatous inflammation and fibrosis, leading to a check-valve mechanism ${ }^{9}$. Regarding the pathogenesis of cystic changes in infectious lung disease such as Pneumocystisjirovecii pneumonia in HIV-infected patients and staphylococcal pneumonia, a check-valve mechanism and excavation of necrotic material via draining bronchi play an important role in forming cystic lesions ${ }^{10,11}$.

Therefore, cystic changes in the present case might have been caused by transbronchial discharge of necrotic material and granulomatous involvement and fibrosis of the bronchioles, leading to a check-valve mechanism.

In conclusion, multiple cystic lung lesions that enlarge after treatment are a rare manifestation of pulmonary cryptococcosis. Even if infection control of cryptococcosis is achieved after the initiation of treatment, 
clinicians should continue to observe the patients carefully.

\section{ACKNOWLEDGMENTS}

None.

\section{CONFLICT OF INTEREST}

None declared.

\section{AUTHOR CONTRIBUTION}

TT: contributed to write-up of abstract, introduction, case presentation, discussion, table and images.

$\mathrm{TN}$; reviewed and edited the manuscript.

\section{ETHICS APPROVAL AND CONSENT TO PARTICIPATE}

Ehics approval was not necessary for this case report. The patient's data and images are de-identified.

INFORMED CONSENT

Written informed consent was obtained from the patient for publication of this case report .

\section{References}

1. Xie LX, Chen YS, Liu SY. Pulmonary cryptococcosis:comparison of CT findings in immunocompetent and immunocompromised patients. Acta Radiol .2015;56:447-453.

2. Woodring JH,Ciporkin G,Lee C, Worm B, Woolley S. Pulmonary cryptoccosis. Semin Roentgenol1996;31:67-75

3. Thitiporn Suwatanapongched, Wasinan Sangsatra, Viboon Boonsarngsuk, Shiriorn P Watcharananan, Pimpin Incharoen. Clinical and radiologic manifestations of pulmonary cryptococcosis in immunocompetent patients and their outcomes after treatment. Diagn Interv Radiol 2013; 19:438-446.

4. Khoury MB, Godwin JD, Ravin CE, Gallis HA, Halvorsen RA, Putman C. Thoracic cryptococcosis: immunologic competence and radiologic appearance. AJR Am. J. Roentgenol. 142:893-896,1984.

5. Noriyo Y, Fumikazu S, Tamiko T, Ishikawa S, Takaki Y,Hishima T, Kamata N. Pulomonay Cryptococcus in rheumatoid arthritis patients:Comparison of imaging characteristics among RA, acquired immunodeficiency syndrome, and immunocompetent patients. European Journal of Radiology 82:20352042,2013

6. Hansell DM, Bankier AA, MacMahon H,McLound TC, Muller NL, Remy J . Fleischner Society: glossary of terms for thoracic imaging. Radiology 246: 697-722,2008.

7. Seiichi T, Yuichi Y, Hitomichi N, Kazuo K, and Etsuo K. A case of secondary pulmonary criptococcosis presenting with multiple cystic shadows. Nihon Kokyuki Gakkai Zasshi 49:315-320,2011(in Japanese)

8. DeMartino E, Go RS, Vassallo R. Langerhans cell histiocytosis and other histiocytic diseases of the lung. Clin Chest Med.37(3):421-430,2016.

9. Kanbouchner M, Basset F, Marchal J, Uhl JF, Hance AJ, Soler P. Three-dimensional Langerhans cell histiocytosis. Am J Respir Crit Care Med 166:1483-1490,2002.

10. Boisset GF. Subpleural emphysema complicating staphylococcal and other pneumonia. J Pediatr.81:259-266,1972.

11. Sandhu JS, Goodman PC. Pulmonary cysts associated with Pneumocystis

carinii pneumonia in patients with AIDS. Radiology 173:33-35,1989

\section{Figure Legends}

Computed tomography showed multiple cavities in the right lung (Fig. 1A).

The lesions coalesced together and their walls became thin (Fig. 1B).

Six months after the initiation of treatment, multiple cysts developped. (Fig 1C). 


\section{Hosted file}

Table(Cryptococcus).pdf available at https://authorea.com/users/385969/articles/522263multiple-cystic-lesions-after-treatment-for-pulmonary-cryptococcosis

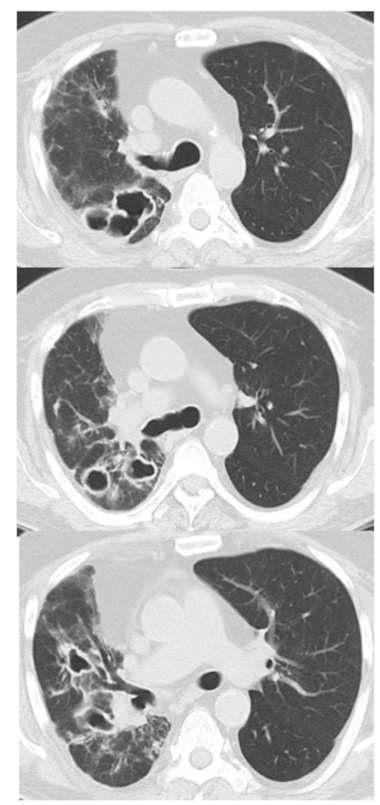

A

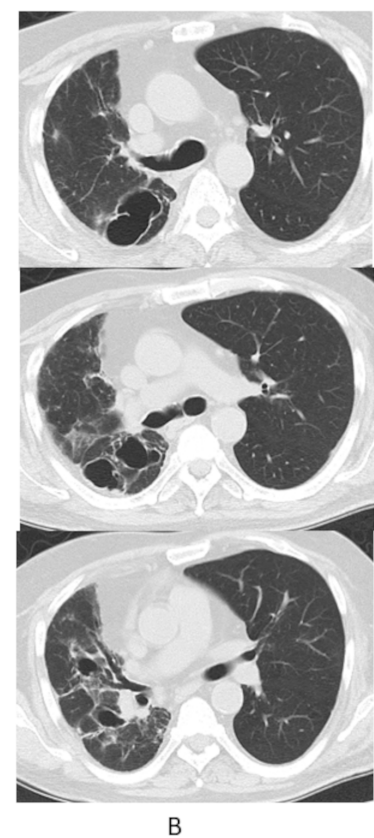

B

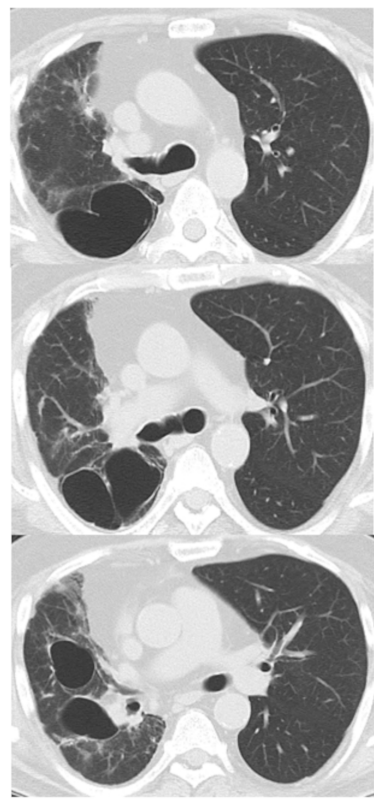

C 